\section{Jugend Sucht: Ein Präventionsbuch - Ehemals Abhängige berichten}

Christoph Möller. Jugend Sucht: Ein Präventionsbuch - Ehemals Abhängige berichten. Stuttgart: Kohlhammer 2019. 143 Seiten, 20,00 Euro, ISBN 978-317-036-559-9

Christoph Möller legt mit der 5. Auflage seines mittlerweile zum Standardwerk gewordenen Büchleins eine komplette neue Überarbeitung und Aktualisierung vor. Während sich die grundlegenden Themen der Suchtentstehung und der gesellschaftlichen sowie individuellen Probleme bei Jugendlichen mit Suchterkrankungen konstant darstellen, gibt es in der Verfügbarkeit, den Zubereitungen, den Dosierungen und vielen anderen Bereichen bei den stoffgebundenen Süchten Veränderungen, auf die der Autor detailliert eingeht. Diese scheinbar abstrakten Veränderungen werden durch die hochinteressanten und prägnanten kurzen Fallgeschichten für den Leser erläutert und greifbar gemacht.

Die zunehmende Bedeutung der stoffungebundenen Verhaltenssüchte und hier insbesondere der Suchterkrankungen im Bereich der modernen Medien werden von Möller ebenfalls anhand typischer Fallbeispiele dargestellt und in ihrer aktuellen und zukünftigen Bedeutung - auch unter klassifikatorischem Aspekt der ICD-11 - erarbeitet. Dem gesamten Werk ist wie schon im Vorwort von Rainer Thomasius angedeutet, eine typische entwicklungspsychiatrische und jugendpsychiatrische Grundhaltung zu eigen, die weder Gefahren dramatisiert oder überbetont noch diese bagatellisiert und sich jeweils am Konzept der Entwicklungsaufgaben und der Entwicklungspsychopathologie orientiert. Dies heisst, dass gerade anhand der eindeutigen Fallbeispiele das individuelle Schicksal und die indivi- duellen Ressourcen für die differenzialdiagnostische Einordnung und die Intervention zentral sind - und nicht abstrakte Gefährlichkeits-Annahmen über Substanzen oder Verhaltensweisen.

Man merkt auch dieser 5. Auflage wieder an, dass die vieljährige Erfahrung der Spezialstation "Teen spirit Island" am Kinderkrankenhaus auf der Bult in Hannover die Grundlage für das Buch liefern und sicher auch in Zukunft liefern werden. Auch diesem Band dürfte eine weite Verbreitung und gute Akzeptanz sicher sein, es ist gerade als Einführungslektüre in diese Thematik auch interdisziplinär gut nutzbar.

Oliver Bilke-Hentsch, Luzern

\section{Physiotherapie bei Kopfschmerzen und Migräne}

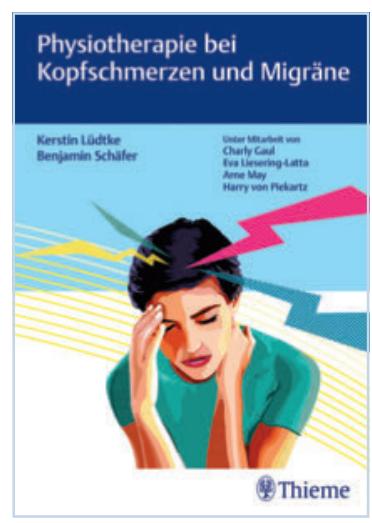

Kerstin Lüdtke, Benjamin Schäfer (Hrsg). Physiotherapie bei Kopfschmerzen und Migräne. Stuttgart: Thieme Verlag 2020. 176 Seiten, 70 Abbildungen, 49,99Euro, ISBN 978-3-13242105-9

Eine seriöse Monografie über den spezifischen Einsatz von Physiotherapie bei Kopfschmerzerkrankungen musste bislang ver- misst werden. Somit füllt das vorliegende Buch eine Lücke in diesem Bereich. Es ist von Physiotherapeuten für Physiotherapeuten konzipiert, enthält aber auch aktuelle Abschnitte über die moderne Diagnostik und leitliniengerechte Therapie von Kopfschmerzerkrankungen aus ärztlicher Sicht.

Am Anfang werden die wichtigsten Kopfschmerzerkrankungen vorgestellt und erläutert. Danach werden die spezifischen anamnestischen und körperlichen Untersuchungsverfahren für Physiotherapeuten dargestellt. Dies schließt auch ein Kapitel über Fragebögen in der Kopfschmerzevaluation mit ein. Im dritten Teil werden die leitliniengerechten Therapieverfahren und die darüberhinausgehenden Therapiemöglichkeiten vorgestellt, dies sowohl in Bezug auf die Pharmakotherapie als auch auf die nicht medikamentösen Verfahren. Dieses Kapitel gliedert sich nach den einzelnen Kopfschmerzarten. Danach werden die verschiedenen Behandlungsansätze im Detail erläutert ohne spezifischen Bezug zu einer speziellen Kopfschmerzerkrankung. Hierbei wird der gesamte Bereich von manueller Therapie bis hin zur multimodalen Schmerztherapie abgedeckt. Schließlich werden noch Anleitungen zur Patientenedukation gegeben mit Abbildungen, die gut im Alltag eingesetzt werden kann.

Wenn man bedenkt, auf welch dünnem Eis man sich manchmal bewegt, wenn man aus wissenschaftlicher Sicht über Physiotherapie und andere nicht medikamentöse Therapieverfahren reden möchte, so hebt sich dieses Buch deutlich davon ab, indem es die praktische Erfahrung der Autoren verknüpft mit dem, was evidenzbasiert ist und mit dem was aus wissenschaftlicher Sicht sinnvoll ist. Dieses Buch schließt in der Tat eine Lücke und sollte weiter Verbreitung finden.

Stefan Evers, Coppenbrügge 